\title{
Educación inicial y brecha digital en pandemia: Estudio en la Provincia Constitucional del Callao
}

Initial education and digital gap in pandemic: Study in the Constitutional Province of Callao

Educação inicial e lacuna digital na pandemia: Estudo na Província Constitucional de Callao

\section{ARTÍCULO GENERAL}

\section{Marleni Mamani Sanchez \\ mmamanis@ucvvirtual.edu.pe}

https://0000-0002-1458-6463

Recibido 25 de Diciembre 2021 | Arbitrado y aceptado 25 de Diciembre 2021 | Publicado el 28 de Enero 2022

\section{RESUMEN}

El objetivo del presente estudio fue analizar la educación inicial considerando la brecha digital en tiempos de pandemia en la provincia constitucional de Callao. Se tuvo un enfoque mixto, de corte transversal y diseño no experimental. Como parte de las técnicas de recolección de datos se utilizaron dos cuestionarios y dos entrevistas. Se contó con la participación de 377 padres y madres y 334 docentes que formaron parte de la comunidad de los centros educativos de educación inicial en la provincia constitucional del Callao. En las entrevistas participaron 24 padres de familia y 37 docentes. Para el procesamiento de la información se utilizó el programa Excel. Los resultados indicaron: un $41.64 \%$ de padres no tenían conocimiento de cómo se utilizan las herramientas digitales; y un $13.47 \%$ de los docentes expresó lo mismo. Una de las principales conclusiones gira entorno a que los docentes desarrollaron estrategias de soporte o acompañamiento a las familias, reconocen y aprovechan el rol activo de los padres en los aprendizajes de sus hijos. Y asumieron con compromiso el reto de desarrollar las clases en línea para niños y niñas de educación inicial, actuando desde su realidad y adaptándose a los recursos con los que contaba.

Palabras claves: Educación inicial; brecha digital; padres de familia; docentes.

\section{ABSTRACT}

The objective of this study was to analyze initial education considering the digital divide in times of pandemic in the constitutional province of Callao. A mixed, cross-sectional and non-experimental design approach was used. As part of the data collection techniques, two questionnaires and two interviews were used. There was the participation of 377 fathers and mothers and 334 teachers who were part of the community of early education educational centers in the constitutional province of Callao. 24 parents and 37 teachers participated in the interviews. For data processing, the Excel program was used. The results indicated: $41.64 \%$ of parents had no knowledge of how digital tools are used; and $13.47 \%$ of teachers expressed the same. One of the main conclusions revolves around the fact that teachers developed support or accompaniment strategies for families, recognize and take advantage of the active role of parents in their children's learning. And they took on the challenge of developing online classes for children in initial education with commitment, acting from their reality and adapting to the resources they had.

Keywords: Initial education; digital divide; parents; teachers.

\section{RESUMO}

O objetivo deste estudo foi analisar a educação inicial considerando a exclusão digital em tempos de pandemia na província constitucional de Callao. Foi utilizada uma abordagem de desenho misto, transversal e não experimental. Como parte das técnicas de coleta de dados, foram utilizados dois questionários e duas entrevistas. Houve a participação de 377 pais e mães e 334 professores que faziam parte da comunidade de centros educativos de educação infantil na província constitucional de Callao. Participaram das entrevistas 24 pais e 37 professores. Para o tratamento dos dados, foi utilizado o programa Excel. Os resultados indicaram: $41,64 \%$ dos pais não tinham conhecimento de como as ferramentas digitais são utilizadas; e $13,47 \%$ dos professores expressaram o mesmo. Uma das principais conclusões gira em torno do facto de os professores terem desenvolvido estratégias de apoio ou acompanhamento às famílias, reconhecendo e aproveitando o papel ativo dos pais na aprendizagem dos seus filhos. E assumiram o desafio de desenvolver aulas online para crianças da educação inicial com comprometimento, atuando a partir de sua realidade e adaptando-se aos recursos que possuíam. Palavras-chave: Educação inicial; brecha digital; pais de família; professores. 


\section{Introducción}

La importancia que adquirió la educación en línea durante el año 2020 es un hecho impensable que marcará un antes y un después en las prácticas pedagógicas y en los sistemas educativos en todo el mundo. Esta situación ha puesto en evidencia las desigualdades sociales, culturales y económicas que viven las familias en más de 180 países víctimas de la pandemia por COVID-19 (Bravo-García \& Magis-Rodríguez, 2020).

La presencia del coronavirus significa un cambio drástico en la forma en como la educación es impartida, ahora el hogar se ha convertido en el único espacio posible para realizar esta actividad, se han modificado los roles y se asumido un nivel de responsabilidad un tanto distinta sobre todo en educación inicial, esto atendiendo a la preocupación de evitar los riesgos al contagio en esta pandemia (García, 2020), este desafío solo es posible gracias a la tecnología (Almario, 2015). El reto de los sistemas educativos a nivel global durante el 2020 fue mantener la vitalidad de la educación y promoción y el desarrollo de aprendizajes significativos (Bonilla \& Guachamín, 2020)

La desigualdad en el acceso a oportunidades educativas por la vía digital incrementa las brechas preexistentes en materia de acceso a la información y el conocimiento, situación que trascendió durante la pandemia y ha tenido su impacto en la educación inicial, dado que más allá del proceso de aprendizaje que se está tratando de impulsar a través de la educación a distancia, el que los niños perdieran contacto con su entorno escolar y los padres de familia a su vez no interactuaran con los docentes acrecentó las dificultades relacionadas con la nueva modalidad de clases en línea, viéndose afectada la socialización y la inclusión en general (Quintana, 2019). De aquí la motivación para realizar este estudio y reconocer con mayor proximidad los desafíos de los padres de familia y docentes de educación inicial durante la pandemia y como la existencia de brechas digitales surgieron y fueron enfrentadas para poder desarrollar el año escolar 2020. Por esta razón es preciso analizar estas brechas desde una perspectiva multidimensional, porque no se trata solo de una diferencia de acceso a equipamiento, sino también del conjunto de habilidades que se requieren para poder aprovechar esta oportunidad, que son desiguales en este caso entre docentes y padres de familia a cargo del cuidado y la mediación del aprendizaje que hoy se realiza desde casa (Martínez, 2018a) 


\section{Aspectos teóricos}

De los 17 objetivos globales de la ONU, el ODS 4 menciona que "la educación permite la movilidad socioeconómica ascendente y es clave para salir de la pobreza" (UNESCO, 2017), por lo cual educar desde la infancia representa la oportunidad para los grupos vulnerables (Tarabini -Castellani, 2020).

En diciembre de 2019 se reportaron una serie de casos de pacientes hospitalizados con una enfermedad nueva caracterizada por neumonía e insuficiencia respiratoria, a causa de un nuevo coronavirus (SARS-CoV-2), en la provincia de Hubei, China (Ferrer, 2020).

Posterior a ello, la Organización Mundial de la Salud (OMS), a los 11 días del mes de marzo del año 2020, declara que el coronavirus COVID-19 pasa de ser una epidemia a ser una pandemia, entre las acciones del Gobierno peruano está la declaración del estado de emergencia el 15 de marzo del 2020 por parte de la presidencia donde se declara la cuarentena.

Esta pandemia trajo consigo no solo millones de muertes alrededor del mundo y consecuencias indirectas como ajustes en las rutinas, separación de amigos y familiares, pérdida de empleos y aislamiento social (Martínez-Taboas, 2020). Lo cual generó el interés de estudiosos de todo el mundo a hacer análisis sobre los impactos sociales que tendría la crisis: concretamente en aspectos como la fragilidad de lo humano en el contexto de la pandemia (De Sousa Santos, 2020), crecimiento del estado de temor al otro (Agamben, 2020), apatía hacia la realidad (Byung-Chul Han, 2020), agitación y stress (Bifo, 2020); racismo, y violencias contra las mujeres (Butler, 2020), condiciones de igualdad para la población (Galindo, 2020) y una reconversión del entramado social existente (Bonilla, 2020) todos estos puntos al final estriban en una marcada fragilización de los servicios públicos, y la educación se encuentra entre ellos(Gentili, 2020); quedando de esta forma expuesta la problemática de como la pandemia no solo afecta directamente la calidad de la educación sino además como esta al ser desarrollada a través de las TIC marca la desigualdad y las condiciones de vulnerabilidad de muchos.

Como parte de una realidad y a medida que la pandemia de la Covid-19 se propagaba por todo el planeta los países anunciaron el cierre temporal de los servicios educativos, lo que afectó a más del 91\% de los estudiantes en el mundo (Morán, 2020). Y en abril de 2020, cerca de 1,600 millones de niños y jóvenes estaban fuera de la escuela (Calderón et al, 2020). 
El cierre de las escuelas supuso pérdida de aprendizajes, aumento en la cantidad de deserciones escolares y mayor inequidad, así como la necesidad de abordar la educación desde otros ámbitos donde la presencialidad no era posible. Debido a ello resurgen la problemática de las brechas de acceso y aprendizaje las cuales, al aumentar, refuerzan el ciclo intergeneracional de pobreza (Gianini \&Grant,2020). Dado este panorama es que las plataformas digitales se convierten en el principal medio de aprendizaje (Prado, 2020) y de hecho la pandemia nos ha hecho aprender que se necesita reunir los recursos educativos en función de garantizar oportunidad para la mayor cantidad de niños posibles, utilizar WhatsApp la cual es una herramienta mucho más popular entre los padres de familia, para ofrecer asistencia pedagógica es una oportunidad para la inclusión de mayor cantidad de niños en educación inicial y sus padres (Cobo \& Hawkins 2020).

En ese sentido, y para dar respuesta a este tipo de situaciones como menciona Cueva et al. (2019), se torna fundamental la capacitación docente. Y pese al contexto de la pandemia los principios generales de la pedagogía siguen siendo válidos a través del aprendizaje remoto, pero existen desafíos adicionales que enfrentan maestros, estudiantes y padres de familia (McAleavy \& Gorgen, 2020).

Al usar la tecnología en el sistema educativo de acuerdo con, Alva (2015) uno de los desafíos a superar esta vinculado a la forma en la que las personas aprovechan al máximo las TIC y las ponen en práctica. Hoy el coronavirus obliga a todos los docentes a utilizar la tecnología, la pandemia ha sido un gran desafío para todas las actividades vinculadas a servicios educativos (Nina \& Frederico,2020). Para Marín (2019) la formación a distancia debe centrarse en el estudiante y adecuar el currículo en función de sus necesidades. Por su parte Escobar \& Gómez (2020) exponen que el uso de herramientas tecnológicas permite desarrollar habilidades comunicativas, fomenta el autoaprendizaje de manera creativa y mejora la comunicación con el docente. Las Tecnologías de la Información y Comunicación (TIC), poco a poco se han convertido en un apoyo dentro de las aulas de clase (Navarrete et al, 2019), la era digital ha sufrido muchas transformaciones, ha apoyado el cambio de los modelos pedagógicos permitiendo que los maestros cambien las metodologías de trabajo y utilicen las tecnologías para la ejecución de procesos educativos innovadores (Vegas, 2020). Para De Moya \& Cózar (2013), citados por Roblizo \& Cózar (2015) las (TIC) se han convertido en un fenómeno social, presente en diferentes ámbitos de la vida humana; trabajo, formación, consumo y diversión estando con ello expuestos a un ambiente virtual en constante cambio. 
Sin embargo, aun con la existencia de las TIC, así como una mejora de las condiciones de acceso entre otros factores es válido mencionar que la pandemia ha resucitado el tema de las brechas digitales, ya que no es cierto que por contar con internet $5 \mathrm{G}$ en el país sus habitantes cuenten con la oportunidad de conexión al más alto nivel y se resuelvan la accesibilidad a las plataformas educativas.

Se denomina brecha digital, como un desfase generacional en el acceso a las nuevas tecnologías. Chacón et al. (2017) y puede es entendida como la desigualdad del acceso a internet y a las tecnologías de la información, lo cual se ha convertido en infraestructura educativa imprescindible para la educación virtual y garantizar el derecho al aprendizaje. De acuerdo al estudio de Hincapié, López-Boo \& Rubio-Codina (2020) se estima que en América Latina en educación primaria el 58\% de los centros urbanos tiene internet, pero en zonas rurales disminuye al 19\%. Razón por la cual es evidente que los sistemas educativos del continente están muy lejos de la disponibilidad de plataformas en línea que aporten al aprendizaje de las y los estudiantes (Moreno \& Gortázar, 2020). Igualmente, se ha reconocido que la disminución de las brechas digitales es el motor para la gestión de conocimientos y desarrollo de competencias generales para el ámbito laboral (Valverde-Berrocoso \& Balladares, 2017). Por tanto, en la medida que se estudie el tema de las brechas digitales se estará analizando las diferencias de accesibilidad y conectividad a internet las cuales crean desigualdad de oportunidades y desarrollo en la población, y está directamente asociado con la combinación de factores socioeconómicos y tecnológicos (Flores, Hernández \&Garay, 2020). Para un mejor análisis de lo que implica una brecha digital es necesario tener claro las diferencias entre áreas urbanas y rurales, problemas de inclusión de personas, diferencias de accesibilidad digital y brechas de género en la educación (Mendoza \& Caldera, 2014; Souza et al., 2017; Jiménez y Fernández, 2016). A esto se suma lo expuesto por Venturini (2020), y Bielschowsky \&Torres (2018), para quienes las brechas digitales están asociadas a conectividad, tipo de dispositivos, apropiación de la tecnología y costo de los servicios. Y aun cuando en los últimos años ha ocurrido una masificación de la conectividad sobre la base del Internet móvil y el incremento de dispositivos digitales más accesibles, aún persisten las brechas (Trucco \& Palma, 2020).

El lograr la incorporación de los recursos tecnológicos en los procesos educativos durante la pandemia, facilita el desarrollo de habilidades específicas en los estudiantes y esto no escapa para los niños en educación inicial (Hurtado, 2020). Por otro lado, se ha convertido en una carrera de obstáculos para el gobierno garantizar el acceso de recursos 
digitales a los estudiantes (Cueto \& León,2020). Esta situación no es del todo nueva ya que de acuerdo a Servindi (2020), sólo 4 de cada 10 hogares en las zonas rurales contaban con acceso a internet a fines del 2019. Por otro lado, según la Encuesta Nacional a Docentes 2018 en Perú (Molina, 2020), 3 de cada 10 profesores de zona rural no cuentan con una computadora y acceso a internet. Esto representa la existencia de estudiantes y docentes desconectados. La puesta en marcha de la tecnología digital como nuevo recurso educativo significa que ahora el ministerio de educación tenga un nuevo problema vinculado a la brecha digital y son las condiciones de la escuela a nivel urbano y rural (Gaudin \& Pareyón, 2020). En Perú según la encuesta del Ministeriode educación (Bustamante, 2020) realizada a padres de familia sobre el medio por el cual acceden al programa "Aprendo en Casa", 18\% lo hace por internet. Para hacerle frente a la problemática acontecida en la actualidad no solo los docentes, sino también los Padres de familia, deberían estar dispuestos a realizar mejoras en los modelos pedagógicos para que esta situación que se dio en la emergencia se traduzca en un cambio a nivel educativo que perdure (Kemelmajer, 2020).

\section{Metodología}

Enfoque y Tipo de investigación: La investigación se ha desarrollado bajo el enfoque mixto el cual es un proceso que recolecta, analiza y vincula datos cuantitativos y cualitativos en una misma investigación (Hernández \& Baptista, 2018). La investigación es de corte transversal, se analizan datos de variables recopiladas en un período de tiempo sobre una población y muestra predefinido.

Diseño de investigación: El diseño es no experimental, se basa fundamentalmente en la observación de fenómenos tal y como se dan en su contexto natural para analizarlos con posterioridad (Hernández \& Baptista, 2018).

Técnicas e instrumentos utilizados: Se ha trabajó con técnicas cuantitativas y cualitativas como es el cuestionario y la entrevista. Ambos aplicados tanto a padres de familia y docentes.

\section{Participantes}

Tabla 1. Población de estudio

\begin{tabular}{lll}
\hline Participantes & Públicas & Privadas \\
\hline Docentes & 241 & 136 \\
\hline Padres de familia & 151 & 183 \\
\hline Total & 377 & 334 \\
\hline
\end{tabular}


Siendo la fórmula de la muestra:

$$
n=\frac{N * Z^{2} * P * Q}{e^{2} *(N-1)+Z^{2} * P * Q}
$$

Docentes la muestra 334 y Padres y madres de familia 337

\section{Procedimientos y análisis de los datos}

La información acopiada, se sometió a un proceso de codificación de datos. Los procedimientos estadísticos por aplicar estuvieron en función del tipo, diseño y nivel de investigación, el procesamiento de información y tabulación se realizó en Excel.

Limitaciones del estudio: Aun cuando el estudio se realizó utilizando las TIC y alcanzó los objetivos planteados, se sugiere hacer nuevos estudios para retomar la temática y profundizar en otras variables asociadas a la misma.

\section{Resultados}

Tabla 2. ¿Conoce usted el término herramienta tecnológica?

\begin{tabular}{|c|c|c|c|c|c|c|c|c|c|c|}
\hline \multicolumn{5}{|c|}{ Padres de familia } & \multicolumn{6}{|c|}{ Docentes } \\
\hline Si & No & & Total & & $\mathrm{Si}$ & & No & & Total & \\
\hline Frec $\%$ & Frec & $\%$ & Frec & $\%$ & Frec & $\%$ & Frec & $\%$ & Frec & $\%$ \\
\hline 50.40 & 187 & 49.60 & 377 & 100 & 329 & 98.50 & 5 & 1.50 & 334 & 88.59 \\
\hline
\end{tabular}

Los docentes expresaron en un $98.50 \%$ que si conocían el término y solo un $1.50 \%$ mencionaron que no, en el caso de los padres de familia los resultados mostraron que un $50.40 \%$ si conocía del término y un $49.60 \%$ no, por lo cual es notorio que los padres de familia se encontraban en desventaja al momento de que inicia la pandemia y las clases entran a un proceso de virtualidad. Situación que ha sido confirmada a través de las entrevistas donde son los docentes quienes se encuentran en mejor posición que los padres en ese tema pero que incluso tuvieron que poner en práctica las herramientas que tenían para el desarrollo de las clases.

Tabla 3. ¿Qué herramienta tecnológica utiliza frecuentemente (Padres de familia)?

\begin{tabular}{|c|c|c|c|c|c|c|c|c|}
\hline & \multicolumn{2}{|c|}{ Internet } & \multicolumn{2}{|c|}{ Facebook } & \multicolumn{2}{|c|}{ WhastApp } & \multicolumn{2}{|c|}{$\begin{array}{l}\text { Correo } \\
\text { electrónico }\end{array}$} \\
\hline & Frec & $\%$ & Frec & $\%$ & Frec & $\%$ & Frec & $\%$ \\
\hline Padres de fan & 354 & 93.90 & 312 & 82.76 & 369 & 97.88 & 41 & 10.88 \\
\hline Docentes & 312 & 93.41 & 330 & 98.80 & 334 & 100 & 215 & 64.37 \\
\hline
\end{tabular}


En las herramientas tecnológicas utilizadas por padres de familia y por docentes los resultados expresan que en un $97.88 \%$ y un $100 \%$ respectivamente es el WhatsApp la herramienta más usada y la que se reconoció incluso como la más sencilla y donde se puede además de hacer llamadas, video llamadas, el envío de documentos, fotos y demás. A través de las entrevistas, padres de familia y docentes externan que para ellos el WhatsApp fue una forma de desarrollar la comunicación, comprender mejor la lógica de las clases en línea, el WhatsApp se convirtió en una base para trazar la estrategia de aprendizaje en línea.

Entrevistas a padres de familia, en este proceso participaron vía Zoom solamente 24 padres de familia

Tabla 4. Entrevista a padres de familia

\section{¿Cómo asumió usted el que las clases fueran en línea durante la pandemia?}

Fueron momentos difíciles porque no se comprendía bien el funcionamiento de las clases en línea para con los niños pequeños, ya que teníamos que dedicar tiempo para organizarnos dentro de casa y apoyar el desarrollo de las clases.

\section{¿Cómo asumió usted el que las clases fueran en línea durante la pandemia?}

Fue realmente algo nuevo, al inicio estábamos muy preocupados, pensamos que nuestros hijos perderían el año escolar, incluso vimos como una carga de trabajo extra para nosotros como padres de familia, porque teníamos que estar presentes durante las clases o al menos los primeros días para comprender como funcionaban.

En el proceso de la entrevista se desarrolló la misma vía zoom contando con la participación de 37 docentes

Tabla 5. Entrevista

\section{¿Qué estrategias utilizó para el desarrollo de las clases en línea?}

Creo que algunas surgieron por el tema de la pandemia, pero básicamente fue la exposición con láminas, juegos, artes escénicas sobre todo para mantener a los niños interesados y que sientan el deseo de conectarse. Se promovió también la comunicación vía plataforma entre sus compañeritos el que los niños se vieran por cámara creaba un sentido de pertenencia en el grupo lo cual ayudó mucho, porque en algunos casos los niños estaban estresados de estar encerrados y ellos no comprendían exactamente qué pasaba porque habían dejado de ir a clases 
¿Cuáles fueron los mayores aprendizajes durante el año 2020 que ha considerado para este nuevo año lectivo?

Se valoró mucho más la labor docente que hacemos cada día por parte de los padres y por nosotros mismos, porque la mayoría tuvimos que reinventarnos para salir adelante. No estábamos preparados para enseñar en línea nos ha tocado aprender en el camino y la verdad es que existe brecha digital entre nosotros mismos y creemos que también eso pasa entre los padres de familia, los cuales se han esforzado también para que esto funcione.

\section{Discusión de resultados}

Sobre el conocimiento de las herramientas digitales previas a la Pandemia Cueva et al. (2019) menciona que es fundamental la capacitación a padres de familia y docentes, esto es reforzado por Kemelmajer (2020) quien precisa se debe estar dispuestos a ajustar los modelos tradicionales y esto es posible al utilizar las tecnologías.

Según Flores, Hernández y Garay (2020) la existencia de brechas digitales se encuentra asociada a accesibilidad y conectividad al internet, lo que genera desigualdad y está asociado con elementos sociales y económicos, esto es respaldado por Mendoza y Caldera (2014), Souza et al., (2017); Jiménez y Fernández (2016). Y de acuerdo con Venturini (2020), y Bielschowsky y Torres (2018), las brechas digitales igualmente se relacionan a diversos factores, tales como; tipo de dispositivos, apropiación de la tecnología y costo de los servicios.

De acuerdo a lo expuesto por Trucco \& Palma (2020), en los últimos años, ha ocurrido un incremento en la adquisición de tecnología móvil y eso hace pensar que se acorta la brecha digital. Sin embargo, según parte Cueto \& León (2020) pese a esta situación debe de priorizarse el acceso a la tecnología a grupos vulnerables.

Para Escobar \& Gómez (2020) en la medida que se creen las condiciones para superar las brechas digitales, se genera la oportunidad de acceder a la educación en línea en condiciones de igualdad lo cual está asociado con lo expresado por Alva (2015), para quien el uso de las TIC favorece el desarrollo de habilidades en distintos ámbitos.

De acuerdo a Vegas (2020) es fundamental la creación de infraestructura digital para el desarrollo de procesos educativos innovadores, así como el acceso a las TIC de 
forma tal que respalde el desarrollo de la educación en línea, y según Prado (2020) esto representa un verdadero desafío para las autoridades y mucho más en el contexto actual de la Pandemia.

\section{Conclusiones}

Durante la pandemia lo que ha agilizado el aprendizaje en línea en la educación inicial es el acompañamiento por parte de los familiares hacia los niños, lo que garantizó que el esfuerzo sostenido de los docentes tenga éxito.

Es necesario el fortalecimiento de las capacidades de los docentes, para "aprender a aprender". Para ser formadores de otros adultos (familiares o cuidadores), usando tecnologías al comunicar, brindar información y acompañar a las familias.

Son los docentes sobre los que descansó la responsabilidad de motivar, ser creativos y sobre todo entender la situación que viven las familias de sus estudiantes, evitando estandarizar procesos pedagógicos, proponer acciones más individualizadas y pertinentes a las necesidades reales.

Los docentes desarrollaron estrategias de soporte o acompañamiento a las familias, reconocen y aprovechan el rol activo de los padres en los aprendizajes de sus hijos. Y asumieron con compromiso el reto de desarrollar las clases en línea para niños y niñas de educación inicial, actuando desde su realidad y adaptándose a los recursos con los que contaba en su entorno.

\section{Referencias bibliográficas}

Almario García, O. (2015). Los retos de la sociedad del conocimiento en Colombia. El Espectador

Agamben, G.2020. Contagio, en Varios autores. 2020. Sopa de Wuhan, Editorial ASPO Alva, A. (2015). Los nuevos rostros de la desigualdad en el siglo XXI: la brecha digital. Revista Mexicana de Ciencias Politicas y Sociales, 60(223), 265-286, e-ISSN: 0185-1918. Recuperado de: http://dx.doi.org/10.1016/S0185-1918(15)72138-0

Bielschowsky, R., y Torres, M. (2018). Desarrollo e igualdad: el pensamiento de la CEPAL en su sétimo decenio. Santiago: CEPAL. https://doi.org/10.18356/7a7b820c-e

"Bifo" Berardi, F. 2020. Crónica de la psicodeflación, en Varios autores. 2020. Sopa de Wuhan, Editorial ASPO 
Byung-Chul Han.2020. El capitalismo tiene sus límites, en Varios autores. 2020. Sopa de Wuhan, Editorial ASPO

Bustamante, R. (junio de 2020). Educación en cuarentena: cuando la emergencia se vuelve permanente (segunda parte). GRADE, Grupo de Anàlisis para el desarollo, (5), 1-12. Recuperado de http://www.grade.org.pe/creer/archivos/articulo-5.pd

Bonilla, L. 2020. Video clase. Conversatorio Educación a distancia y Reingeniería Social, Alerta educativa, recuperado de: https://www.youtube.com/watch?v=nZcoKvrcxVY

Bonilla-Guachamín, J. A. (2020). Las dos caras de la educación en el COVID-19. CienciAmérica, 9(2), 89-98. http://dx.doi.org/10.33210/ca.v9i2.294

Bravo-García, E., y Magis-Rodríguez, C. (2020). La respuesta mundial a la epidemia del COVID-19: los primeros tres meses. Boletín sobre COVID-19 Salud Pública y

Epidemiología, $1(1)$ 3-8. http://dsp.facmed.unam.mx/wpcontent/uploads/2013/12/COVID-19-No.1-03-Larespuesta-mundial-a-la-epidemiadel-COVID-19-los-primeros-tres-meses.pdf

Butler, J.2020. La emergencia viral y el mundo de mañana, en Varios autores. 2020. Sopa de Wuhan, Editorial A

Calderón, A-I., y Borges, R. M. (2020). Avaliação em larga escala na Educação Básica: usos e tensões teórico-epistemológicas. Meta: Avaliação, 12(34), 28-56. https://doi.org/10.22347/2175-2753v12i34.2281

Chacón-Penagos, A. M., Ordóñez-Córdoba, J. A., \& Anichiarico-González, A. M. (enerojunio de 2017). Hacia el reconocimiento de la inclusión digital como un derecho fundamental en Colombia. Vniversitas (134), 139-168.

Cobo, C, Hawkins, R, Rovner, H. 2020. Cómo utilizan la tecnología los países de América Latina durante el cierre de las escuelas a causa de la COVID-19

Cueto, S., León, J., \& Felipe, C. (2020). Acceso a dispositivos y habilidades digitales de dos cohortes en el Perú. [Análisis \& Propuestas, 56]. Lima: GRADE; Niños del Milenio.

Cueva, J., García, A., \& Martínez, O. (2019). El conectivismo y las TIC: Un paradigma que impacta el proceso enseñanza aprendizaje. Revista Scientific, 4(14), 205-227, 
e-ISSN:

De Sousa Santos, B. 2020. La cruel pedagogía del virus. Buenos Aires, Argentina: Masa Crítica-CLACSO

Escobar F., y Gómez I. (2020). WhatsApp para el desarrollo de habilidades comunicativas orales y escritas en adolescentes peruanos. Comunicar, 28(65), 111-120. https://doi.org/10.3916/C65-2020-10

Ferrer, R. (2020) Pandemia por COVID-19: el mayor reto de la historia del intensivismo. Medicina Intensiva, 44(6), 323-324.

Flores, J., Hernández, R., y Garay, R. (2020). Tecnologías de información: Acceso a internet y brecha digital en Perú. Revista Venezolana de Gerencia, 25(90). https://doi.org/10.37960/rvg.v25i90.32396

Galindo, M. 2020. Desobediencia, por tu culpa voy a sobrevivir, en Varios autores. 2020. Sopa de Wuhan, Editorial ASPO

García, L. (2020). Bosque semántico: ¿educación/enseñanza/aprendizaje a distancia, virtual, en línea, digital, eLearning...? RIED: Revista Iberoamericana de Educación a Distancia, 23(1), 9-28, e-ISSN: 1390- 3306. Recuperado de: http://dx.doi.org/10.5944/ried.23.1.2549

Gentili, P. 2020. Video clase, Clase magistral: La Educación en América Latina en tiempos de pandemia, Alerta educativa, recuperado de: https://www.youtube.com/watch?v=6uoDXaMPoH8

Giannini, S. y Grant S. 2020. Three ways to plan for equity during the coronavirus school closures, GEM Report blog.

Hincapie, D, Lopez-Boo, F y Rubio-Codina, M. 2020. El alto costo del COVID-19 para los niños. Estrategias para mitigar su impacto en América Latina y el Caribe. Documento para discusión.

Kemelmajer, Cintia (2020). Educación en tiempos de pandemia: consejos de especialistas para enriquecer las aulas virtuales. Recuperado de: https://www.conicet.gov.ar/educacion-entiempos-de-pandemia-consejos-deespecialistas-para-enriquecer-las-aulas-virtuales/ 
Hernández Sampieri, R., Fernández Collado, C., \& Pilar, B. L. (2018). Metodología de la investigación. México: McGraw-Hill.

Hurtado, F. J. (2020) Educación, Sociedad e Ideología: La Trilogía Imperante del Siglo XXI. [Artículo en línea] Revista Arbitrada del Centro de Investigación y Estudios Gerenciales, 42, 138-149 Recuperado de: www.grupocieg.org/archivos_revista/Ed.42(138149)\%20Frank\%20Junior\%20Hurtado\%20Talavera_articulo_id602.pdf

Jiménez, R., \& Fernández, C. (2016). La brecha de género en la educación tecnológica. Ensaio: Avaliação e Políticas Públicas em Educação, 24(92), 743-771. https://doi.org/10.1590/S0104-403620160003000010

Marín, N. (2019). Las Tecnologías de Información y Comunicación: Una Gestión Educativa desde la Plataforma Moodle. Revista Scientific, 4(12), 329-339, e-ISSN: 2542-2987.

https://doi.org/10.29394/Scientific.issn.2542$\underline{2987.2019 .4 .12 .17 .329339}$

Martínez, O. (2018a,b). Uso de las Tecnologías de la Información y la Comunicación en la Educación Básica. Revista Scientific, 3(10), 154- 174, e-ISSN: 2542-2987. Recuperado de: https://doi.org/10.29394/Scientific.issn.25422987.2018.3.10.8.154-174

Martínez-Taboas, A. (2020). Pandemias, COVID-19 y Salud Mental: ¿Qué Sabemos Actualmente? Revista Caribeña de Psicología, 4(2), 143-152. https://doi.org/10.37226/rcp.v4i2.4907

Mendoza, J., y Caldera, J. (2014). Umbrales para la determinación de la brecha digital: comparativa entre regiones desarrolladas. Transinformação, 26(2), 125-132. https://doi.org/10.1590/0103-37862014000200002

Molina, O. (23 de agosto de 2020). El otro lado de la pandemia: las pérdidas en educación. El Comercio. Recuperado de http://bit.ly/2Wo9to

Morán, M. (2020). Educación. Desarrollo Sostenible. https://www.un.org/sustainabledevelopment/es/education/

McAleavy, T y Gorgen, K. 2020. Summary, Overview of emerging country level response to providing educational continuity under COVID-19. Best practice in pedagogy for remote teaching. Part of our EdTech and coronavirus (COVID-19) series: edtechhub.org/coronavirus. 
Moreno, J M y Gortázar, L. 2020. Schools' readiness for digital learning in the eyes of principals. An analysis from PISA 2018 and its implications for the COVID19 (Coronavirus) crisis response

Navarrete, G., Vera, M., \& Idrovo, K. (2019). Un nuevo mundo educativo: organización, funcionamiento y estructura. Una propuesta a la educación ecuatoriana. Espirales: revista multidisciplinaria de investigación, 3(26), 50-59, e-ISSN: 2550-6862. Recuperado de: https://doi.org/10.31876/re.v3i26.459

Nina, M., y Frederico, A. (2020). El rol de las bibliotecas en tiempos de COVID-19: reflexiones y propuestas. Desde el Sur, 241-262. https://doi.org/10.21142/DES1201-2020-001

Prado, E. (29 de octubre de 2020). Hacia la transformación digital del país para remontar la crisis. La República. Perú. Recuperado de http://bit.ly/2LKAvEv

Quintana, J. (2019). Estudio de avances en la implementación del teletrabajo en la Universidad Nacional Abierta y a Distancia UNAD 2016 - 2019. Tesis. Bogotá, Colombia: Universidad Nacional Abierta y a Distancia UNAD. Recuperado de: https://repository.unad.edu.co/handle/10596/3111

Roblizo, M., \& Cózar, R. (2015). Usos y competencias en TIC en los futuros maestros de educación infantil y primaria: hacia una alfabetización real para docentes. PíxelBit: Revista de Medios y Educación, (47), 2339, e-ISSN: 2171-7966. https://recyt.fecyt.es/index.php/pixel/article/view/61669

Servindi. (30 de diciembre de 2020). Brecha digital: el futuro de la próxima generación en riesgo. Recuperado de http://bit.ly/2LLjdY

Souza, C., Siqueira, É., \& Reinhard, N. (2017). Digital divide of small and medium-sized enterprises: an analysis of influencing factors using the toe theory. Ram. Revista de $\begin{array}{lll}\text { Administração } & \text { Mackenzie, } & \text { 15-48. }\end{array}$ https://doi.org/10.1590/167869712017/administracao.v18n2p15-48

Tarabini-Castellani, C. (2020) Educación, pobreza y desarrollo: Agendas globales, políticas nacionales, realidades locales. Barcelona, España: Universitat Autònoma de Barcelona. Departament de Sociología.

Trucco, D. y A. Palma (eds.) (2020). Infancia y adolescencia en la era digital: un informe comparativo de los estudios de Kids Online del Brasil, Chile, Costa Rica y el 
Marleni Mamani Sanchez

Uruguay. Documentos de Proyectos (LC/TS.2020/18), Santiago, Comisión Económica para América Latina y el Caribe (CEPAL).

Unesco. (2017). Educación para los Objetivos de Desarrollo Sostenible: objetivos de aprendizaje.

https://www.researchgate.net/publication/325570670_Educacion_para_los_Objeti vos de Desarrollo_Sostenible Objetivos de aprendizaje

Valverde-Berrocoso, J., \& Balladares, J. (2017). Enfoque sociológico del uso del Blearning en la educación digital del docente universitario. Sophia: colección de Filosofía de la Educación, 23(2), 123-140.

Vegas, M. (15 de diciembre de 2020). Lecciones y desafíos hacia el 2021. El Comercio. Recuperado de http://bit.ly/2LAsPEC

Venturini, J. (27 de marzo de 2020). Los límites de la educación a distancia en América Latina. Recuperado de http://bit.ly/3gWJDS8 